\title{
Tumor size as a prognostic factor improves the accuracy of the prognostic prediction of T4 stage colon cancer: a propensity score analysis
}

\author{
Yuexiang Liang \\ the First Affiliated Hospital of Hainan Medical University \\ Qiang Li \\ the First Affiliated Hospital of Hainan Medical University \\ Donglei He \\ the First Affiliated Hospital of Hainan Medical University \\ Yong Chen \\ the First Affiliated Hospital of Hainan Medical University \\ Jingquan Li ( $\sim$ hnlijingquan@163.com ) \\ the First Affiliated Hospital of Hainan Medical University
}

\section{Research Article}

Keywords: colon cancer, tumor size, T4 stage, prognosis

Posted Date: February 19th, 2021

DOl: https://doi.org/10.21203/rs.3.rs-209184/v1

License: (a) This work is licensed under a Creative Commons Attribution 4.0 International License. Read Full License

Version of Record: A version of this preprint was published at Scientific Reports on August 11th, 2021. See the published version at https://doi.org/10.1038/s41598-021-95828-4. 


\section{Abstract}

The aim of this study was to evaluate the potential impact of tumor size on the long-term outcome of CC patients after curative surgery. A total of 782 curatively resected T4 stage $\mathrm{CC}$ patients without distant metastasis were enrolled. Patients were categorized into 2 groups according to the best threshold of tumor size: larger group (LG) and smaller group (SG). Propensity score matching was used to adjust for the differences in baseline characteristics. The ideal cutoff points for tumor size was $5 \mathrm{~cm}$. In the multivariate analysis for the whole study series, tumor size was an independent prognostic factors. Patients in the LG had a significant lower 5-year OS rate, but higher distant metastatic rate than those in the SG (37.1\% versus $25.2 \%$, $P<0.001)$. After matching, patients in the LG still demonstrated a significant lower 5 -year OS rate than those in the SG $(63.5 \%$ versus $74.2 \%, P<0.001)$. Patients in the LG benefited more from postoperative adjuvant chemotherapy than patients in the SG. The modified stage including tumor size was found to be more appropriate for predicting the OS of T4 stage CC than TNM stage. In conclusion, tumor size was an independent prognostic factor and could correlate with higher distant metastasis rate and better response of adjuvant chemotherapy. We maintain that tumor size should be incorporated into the staging system to enhance the accuracy of the prognostic prediction of T4 stage CC patients.

\section{Introduction}

Tumor size has been verified to be associated with overall survival (OS) in many types of malignancy, and it is regarded as " $T$ " stage of many solid tumors including breast, lung and liver cancers in the tumor-nodemetastasis (TNM) staging system of the Union for International Cancer Control (UICC) ${ }^{1-5}$. Despite the value of tumor size as a prognostic indicator in those solid tumors, the prognostic significance of tumor size in gastrointestinal tumors has not been widely realized. Deng JY et al. ${ }^{6}$ demonstrated that tumor size as a T stage could accurately predict the survival of gastric cancer patients and it was an independent prognostic factor in multivariate analysis. Kunisaki $\mathrm{C}$ et al. ${ }^{7}$ found that tumor size was a reliable prognostic factor of gastric cancer, and thus suggested that it should be included in staging system. As for colon cancer, few studies $^{8-16}$ focused on tumor size. Saha Sukamal et al. ${ }^{8}$ reported that tumor size positively correlated with grade, $T$ stage and node stage, and it was inversely associated with survival.

As the tumor size usually correlated with tumor stage and other prognostic factors as previous studies ${ }^{8-16}$ reported, therefore, it was vital important to adjust for imbalances regarding baseline characteristics between patients with larger tumor size and those with smaller one, especially in retrospective analysis. In the present study, we used both Cox proportional hazard regression analysis and propensity score method to overcome bias due to different distribution of covariates for the groups. Our ultimate aim was to evaluate the potential impact of tumor size on the long-term outcome of CC patients after curative surgery in a single center in China.

\section{Material And Methods}

\section{Patients}


This study was reviewed and approved by the Ethics Committee of the First Affiliated Hospital of Hainan Medical University. All the patients signed an informed consent form for the operation including surgical procedure. All processes involved in this study were in accordance with the standards of the institutional Ethics Committee. A total of 1207 patients with $\mathrm{CC}$ who underwent surgical resection at the First Affiliated Hospital of Hainan Medical University between January 2004 and December 2014 were eligible for this study. Eligibility criteria for this study included: (1) adenocarcinoma of the colon, (2) stage I to IV disease underwent colonectomy plus lymph node dissection with curative intent, (3) patients were completely followed-up, (4) no history of other malignancy, (5) no history of neoadjuvant chemotherapy, (6) no patients died during the initial hospital stay or for 1 month after surgery. Ultimately, 1008 patients were included in this study. The stratified survival analysis of tumor stage reveled that the prognosis of patients in IV and T1-3 stages was not affected by tumor size (Table 1). After excluding 100 patients with distant metastasis and 126 patients without serosa invasion, finally, a total of 782 T4 stage patients without disant metastasis were included in the analysis.

Table 1

Strata survival analysis of the CC patients according to tumor stages.

\begin{tabular}{|c|c|c|c|c|c|c|}
\hline \multirow[t]{2}{*}{ Tumor stage } & \multicolumn{2}{|l|}{ SG } & \multicolumn{2}{|l|}{ LG } & \multirow[t]{2}{*}{$x^{2}$} & \multirow[t]{2}{*}{$P$} \\
\hline & $\mathrm{n}$ & 5-year OS(\%) & $\mathrm{n}$ & 5-year OS(\%) & & \\
\hline \multicolumn{7}{|l|}{ T stage } \\
\hline T2 & 20 & 100.0 & 34 & 91.2 & 0.280 & 0.597 \\
\hline T3 & 23 & 87.0 & 49 & 71.4 & 0.777 & 0.378 \\
\hline $\mathrm{T} 4$ & 316 & 70.8 & 566 & 59.0 & 17.607 & $<0.001$ \\
\hline \multicolumn{7}{|l|}{ TNM stage } \\
\hline I & 16 & 100.0 & 30 & 90.0 & 0.256 & 0.613 \\
\hline II & 214 & 80.6 & 391 & 72.8 & 7.861 & 0.005 \\
\hline III & 99 & 68.4 & 158 & 48.7 & 8.429 & 0.004 \\
\hline IV & 30 & 26.7 & 70 & 17.1 & 3.134 & 0.077 \\
\hline Total & 359 & 73.5 & 649 & 61.7 & 17.707 & $<0.001$ \\
\hline
\end{tabular}

\section{Evaluation of clinicopathological variables and survival}

Clinicopathological features studied included the following 13 factors: sex, age at surgery, tumor location, tumor size, histology, lymphovascular invasion, presence of lymph node metastasis, number of lymph nodes retrieval, surgical procedure, postoperative complications, postoperative chemotherapy, peroperative serum carbohydrate antigen 19 - 9 (CA19-9) level and carcinoembryonic antigen (CEA) level.

All the patients underwent curative colonectomy plus complete mesocolic excision and lymph node dissection. The tumors were staged according to the eighth edition of the UICC TNM classification system. Tumors were classified into two groups based on histology: differentiated type, including papillary, well or 
moderately differentiated adenocarcinoma; and undifferentiated type, including poorly differentiated or undifferentiated adenocarcinoma, signet ring cell carcinoma and mucinous carcinoma.

\section{Mesurement of tumor size}

The resected specimen was opend along the longitudinal axis of the colon wall on the opposite side of the tumor. Then, the opened colon was placed on a flat board with the mucosal side facing up. During the examination from the mucosal side, the longest tumor diameter was measured and regarded as tumor size in this study.

\section{Follow up}

The patients were followed up by his attending physician and the research nurse of our department. To increase the follow-up rate, methods such as telephone, message, correspondence and outpatient department visits were used together. The patients were followed up every 3 months up to 2 years after surgery, then every 6 months up to 5 years, and then every year or until death. Physical examination, laboratory test (including assessing CEA and CA19-9), abdominal ultrasound (US), chest and abdominal computed tomography (CT) were performed at each visit, while endoscopy was obtained every year. The OS rate was calculated from the day of surgery until time of death or final follow-up. The date of final follow-up was December 31, 2019.

\section{Statistical analysis}

For continuous variables, which were presented as mean \pm standard deviation (SD), parametric analysis was performed using Student's t test. Categorical variables were analyzed by means of the chi-square or Fisher's exact test. Overall survival curves were calculated using the Kaplan-Meier method based on the length of time between primary surgical treatment and final follow-up or death. The log-rank test was used to assess statistical differences between curves. Independent prognostic factors were identified by the Cox proportional hazard regression model. To overcome bias due to the different distribution of covariates for the two groups, the propensity score analysis was used to obtain a one-to-one match by using the nearest-neighbor matching method. And we imposed a caliper of 0.25 of the sd of the logit of the propensity score. Variables involved in the propensity model were sex, age at surgery, tumor location, tumor size, histology, lymphovascular invasion, presence of lymph node metastasis, number of lymph nodes retrieval, surgical procedure, postoperative complications, postoperative chemotherapy, peroperative serum CA19-9 level and CEA level. To compare our suggested new modified tumor-size-node-metastasis (mTSNM) stage with the eighth edition of TNM staging system, the -2 log likelihood, hazard ratio (HR) value, and 95\% confidence interval (Cl) related to the Cox regression model were used for measuring homogeneity and discriminatory ability. $P<0.050$ (bilateral) was considered statistically significant. The statistical analysis was performed using the statistical analysis program package SPSS 22.0 (SPSS, Chicago, IL).

\section{Results}

\section{Clinicopathological features and outcome of the whole study series before matching}

The median follow-up for the whole study series was 67 (range: 5-105) months and the 5-year OS rate was $67.8 \%$. Seven hundred and eighty-two patients included 439 males $(56.1 \%)$ and 343 females (43.9\%). The age ranges from 26 to 83 years, and median age was 61 years. Of the 782 patients who underwent curative 
surgery, 597 patients underwent laparoscopic surgery, and the remaining 185 patients underwent open surgery. Of these patients, 601 received postoperative adjuvant chemotherapy with 5-fluorouracil, leucovorin and oxaliplatin (FOLFOX6); capecitabine and oxaliplatin (XELOX); or capecitabine alone.

The mean $\pm S D$ tumor size was $6.17 \pm 2.59 \mathrm{~cm}$ (range $0.80-17.00 \mathrm{~cm}$ ). To identify the optimal cutoff points for tumor size, the cut-point survival analysis was adopted, and survival rates were calculated at each $1-\mathrm{cm}$ interval. The tumor size with the highest $\chi^{2}$ value was regarded as the optimal threshold of classification. After numerous evaluation, the optimal thresholds were determined by the best cutoff approach in terms of the logrank test. The ideal tumor size cutoff value was $5 \mathrm{~cm}$ in this study. The tumor size intervals were $S 1,<5 \mathrm{~cm}$ and $S 2, \geq 5 \mathrm{~cm}$. All the patients were categorized into two groups based on tumor size: the larger group (LG), patients with tumor size $\geq 5 \mathrm{~cm}$, including 496 patients; and the smaller group (SG), patients with tumor size< $5 \mathrm{~cm}$, including 286 patients. Clinicopathologic variables were compared between the two groups as shown in left columns of Table 2. There were no statistical differences in sex, age at surgery, lymph node retrieval $(\geq 12$ $v s<12$ ), lymph node metastasis, preoperative serum CEA level and postoperative adjuvant chemotherapy between the two groups, whereas patients in the LG were more likely to have a larger number of lymph node retrieval $(16.7 \pm 7.5 v s 15.1 \pm 5.6, P=0.001)$, and a higher ratio of tumor located at right colon $(65.3 \% v s 54.5 \%$, $P=0.003)$, but less likely to undergo laparoscopic surgery $(71.4 \%$ vs $85.0 \%, P<0.001)$ than those in the SG. Besides, undifferentiated histology $(39.1 \%$ vs $29.4 \%, P=0.006)$, lymphovascular invasion $(18.1 \%$ vs $11.2 \%, P=$ $0.010)$, elevated CA19-9 (21.8\% vs $13.3 \%, P=0.003)$ and postoperative complications $(10.3 \%$ vs $2.8 \%, P<$ 0.001 ) were more prevalent in larger tumors. 
Table 2

Clinicopathological features of T4 CC patients grouped by tumor size: data are reported for the whole study series and for one-to-one propensity-score matched pairs

\begin{tabular}{|c|c|c|c|c|c|c|}
\hline \multirow[t]{2}{*}{ Characteristics } & \multicolumn{3}{|c|}{ Whole study series } & \multicolumn{3}{|c|}{$\begin{array}{l}\text { Matched pairs (Case-control } \\
\text { Method) }\end{array}$} \\
\hline & $\begin{array}{l}\text { SG(n = } \\
286)\end{array}$ & $\begin{array}{l}\mathrm{LG}(\mathrm{n}= \\
496)\end{array}$ & $\mathbf{P}$ & $\begin{array}{l}\text { SG(n= } \\
286)\end{array}$ & $\begin{array}{l}\mathrm{LG}(\mathrm{n}= \\
286)\end{array}$ & $\mathbf{P}$ \\
\hline Sex & & & 0.118 & & & 0.397 \\
\hline Male/ Female & $171 / 115$ & $268 / 228$ & & $171 / 115$ & $161 / 125$ & \\
\hline Age at surgery (yr) & & & 0.648 & & & 0.485 \\
\hline$\geq 65 /<65$ & $98 / 188$ & $178 / 318$ & & $98 / 188$ & $106 / 180$ & \\
\hline Mean age at surgery & & & 0.087 & & & 0.994 \\
\hline Mean \pm sd & $60.8 \pm 11.1$ & $59.3 \pm 12.7$ & & $60.8 \pm 11.1$ & $60.8 \pm 11.4$ & \\
\hline Tumor location & & & 0.003 & & & 0.448 \\
\hline Right colon/Left colon & $156 / 130$ & $324 / 172$ & & $156 / 130$ & $165 / 121$ & \\
\hline Histology & & & 0.006 & & & 0.260 \\
\hline Differentiated/Undifferentiated & $202 / 84$ & $302 / 194$ & & $202 / 84$ & $214 / 72$ & \\
\hline Lymphovascular invasion & & & 0.010 & & & 0.605 \\
\hline Present / Absent & $32 / 254$ & $90 / 406$ & & $32 / 254$ & $36 / 250$ & \\
\hline $\begin{array}{l}\text { Number of lymph nodes } \\
\text { retrieval }\end{array}$ & & & 0.001 & & & 0.144 \\
\hline Mean \pm sd & $15.1 \pm 5.6$ & $16.7 \pm 7.5$ & & $15.1 \pm 5.6$ & $15.9 \pm 7.2$ & \\
\hline lymph nodes retrieval & & & 0.472 & & & 0.288 \\
\hline$>12 / \leq 12$ & $209 / 77$ & $374 / 122$ & & $209 / 77$ & $220 / 66$ & \\
\hline Lymph node metastasis & & & 0.167 & & & 0.520 \\
\hline N0/N1/N2 & $193 / 67 / 26$ & $354 / 89 / 53$ & & $193 / 67 / 26$ & $198 / 57 / 31$ & \\
\hline CEA level & & & 0.787 & & & 0.556 \\
\hline Elevated / Normal & $124 / 162$ & $220 / 276$ & & $124 / 162$ & $131 / 155$ & \\
\hline CA19-9 level & & & 0.003 & & & 0.406 \\
\hline Elevated / Normal & $38 / 248$ & $108 / 388$ & & $38 / 248$ & $45 / 241$ & \\
\hline Surgical procedure & & & $\begin{array}{l}< \\
0.001\end{array}$ & & & 0.064 \\
\hline Open /Laparoscopic & $43 / 243$ & $142 / 354$ & & $65 / 221$ & $60 / 226$ & \\
\hline Postoperative chemotherapy & 74.1 & 78.4 & 0.170 & & & 0.138 \\
\hline
\end{tabular}




\begin{tabular}{|c|c|c|c|c|c|c|}
\hline \multirow[t]{2}{*}{ Characteristics } & \multicolumn{3}{|c|}{ Whole study series } & \multicolumn{3}{|c|}{$\begin{array}{l}\text { Matched pairs (Case-control } \\
\text { Method) }\end{array}$} \\
\hline & $\begin{array}{l}S G(n= \\
286)\end{array}$ & $\begin{array}{l}\mathrm{LG}(\mathrm{n}= \\
496)\end{array}$ & $\mathbf{P}$ & $\begin{array}{l}S G(n= \\
286)\end{array}$ & $\begin{array}{l}\mathrm{LG}(\mathrm{n}= \\
286)\end{array}$ & $\mathbf{P}$ \\
\hline Yes/No & $212 / 74$ & $389 / 107$ & & $212 / 74$ & $227 / 59$ & \\
\hline Postoperative complications & & & $\begin{array}{l}< \\
0.001\end{array}$ & & & 0.136 \\
\hline Present / Absent & $8 / 278$ & $51 / 445$ & & $8 / 278$ & $15 / 271$ & \\
\hline
\end{tabular}

In the entire study population, patients with larger tumors had a lesser 5-year OS than those with smaller tumors (5-year OS: $63.5 \%$ vs $75.2 \%, P<0.001$, Fig. $1 \mathrm{~A}$ ). The results of the univariate and multivariate survival analyses are presented in Table 3. A total of 12 factors evaluated in the univariate analysis had a significant effect on survival: age at surgery ( $<65 v s \geq 65$ ), tumor location, tumor size ( $<5 v s \geq 5 \mathrm{~cm}$ ), histology, $N$ stage, lymphovascular invasion, lymph node retrieval (>12 vs $\leq 12)$, surgical procedure, CEA level, CA19-9 level, postoperative complications and postoperative chemotherapy. In the multivariate analysis, age at surgery, $\mathrm{N}$ stage, lymphovascular invasion, lymph node retrieval, CA19-9 level, postoperative complications, postoperative chemotherapy and tumor size (HR was 1.433 for $L G, P=0.014$ ) were found to be independent prognostic factors for OS. 
Table 3

Univariate and multivariate survival analysis in the whole study series.

\begin{tabular}{|c|c|c|c|c|c|c|}
\hline \multirow[t]{2}{*}{ Characteristics } & \multirow[t]{2}{*}{$n(\%)$} & \multirow{2}{*}{$\begin{array}{l}\text { 5-year OS } \\
\text { (\%) }\end{array}$} & \multicolumn{2}{|c|}{ Univariate analysis } & \multicolumn{2}{|c|}{ Multivariate analysis } \\
\hline & & & $\mathrm{HR}(95 \% \mathrm{Cl})$ & $\mathbf{P}$ & $\mathrm{HR}(95 \% \mathrm{Cl})$ & $\mathbf{P}$ \\
\hline \multicolumn{7}{|l|}{ Sex } \\
\hline Male & $439(56.1)$ & 67.9 & 1 (ref) & & & \\
\hline Female & $343(43.9)$ & 67.6 & $\begin{array}{l}1.020(0.806- \\
1.290)\end{array}$ & 0.871 & & \\
\hline \multicolumn{7}{|c|}{ Age at surgery (yr) } \\
\hline$<65$ & $506(64.7)$ & 71.1 & 1 (ref) & & 1 (ref) & \\
\hline$\geq 65$ & 276(35.3) & 61.6 & $\begin{array}{l}1.442(1.138- \\
1.828)\end{array}$ & 0.002 & $\begin{array}{l}1.358(1.048- \\
1.759)\end{array}$ & 0.021 \\
\hline \multicolumn{7}{|l|}{$\begin{array}{l}\text { Primary tumor } \\
\text { location }\end{array}$} \\
\hline Right colon & $480(61.4)$ & 63.1 & 1 (ref) & & 1 (ref) & \\
\hline Left colon & $302(38.6)$ & 75.2 & $\begin{array}{l}0.653(0.509- \\
0.839)\end{array}$ & 0.001 & $\begin{array}{l}0.910(0.689- \\
1.201)\end{array}$ & 0.504 \\
\hline \multicolumn{7}{|l|}{ Tumor size } \\
\hline $\mathrm{SG}(<5 \mathrm{~cm})$ & $286(36.6)$ & 75.2 & 1 (ref) & & 1 (ref) & \\
\hline $\mathrm{LG}(\geq 5 \mathrm{~cm})$ & $496(63.4)$ & 63.5 & $\begin{array}{l}1.710(1.318- \\
2.217)\end{array}$ & $<0.001$ & $\begin{array}{l}1.433(1.076- \\
1.908)\end{array}$ & 0.014 \\
\hline \multicolumn{7}{|l|}{ Histology } \\
\hline Differentiated & $504(64.5)$ & 72.4 & 1 (ref) & & 1 (ref) & \\
\hline Undifferentiated & $278(35.5)$ & 59.4 & $\begin{array}{l}1.589(1.256- \\
2.010)\end{array}$ & $<.001$ & $\begin{array}{l}1.238(0.951- \\
1.612)\end{array}$ & 0.112 \\
\hline \multicolumn{7}{|l|}{$\mathrm{N}$ stage } \\
\hline NO & $547(69.9)$ & 75.3 & 1 (ref) & & 1 (ref) & \\
\hline N1 & $156(20.0)$ & 59.0 & $\begin{array}{l}1.835(1.386- \\
2.429)\end{array}$ & $<0.001$ & $\begin{array}{l}2.051(1.531- \\
2.747)\end{array}$ & $\begin{array}{l}< \\
0.001\end{array}$ \\
\hline N2 & $79(10.1)$ & 32.9 & $\begin{array}{l}4.033(2.976- \\
5.465)\end{array}$ & $<.001$ & $\begin{array}{l}4.369(3.192- \\
5.979)\end{array}$ & $<.001$ \\
\hline \multicolumn{7}{|l|}{$\begin{array}{l}\text { Lymphovascular } \\
\text { invasion }\end{array}$} \\
\hline Absent & $660(84.4)$ & 71.1 & 1 (ref) & & 1 (ref) & \\
\hline Present & $122(15.6)$ & 50.0 & $\begin{array}{l}2.209(1.542- \\
2.670)\end{array}$ & $<.001$ & $\begin{array}{l}1.760(1.295- \\
2.392)\end{array}$ & $<.001$ \\
\hline
\end{tabular}




\begin{tabular}{|c|c|c|c|c|c|c|}
\hline \multirow[t]{2}{*}{ Characteristics } & \multirow[t]{2}{*}{ n (\%) } & \multirow{2}{*}{$\begin{array}{l}\text { 5-year OS } \\
\text { (\%) }\end{array}$} & \multicolumn{2}{|c|}{ Univariate analysis } & \multicolumn{2}{|c|}{ Multivariate analysis } \\
\hline & & & $\mathrm{HR}(95 \% \mathrm{Cl})$ & $\mathbf{P}$ & $\mathrm{HR}(95 \% \mathrm{Cl})$ & $\mathbf{P}$ \\
\hline \multicolumn{7}{|c|}{ lymph nodes retrieval } \\
\hline$\leq 12$ & $199(25.4)$ & 58.8 & 1 (ref) & & 1 (ref) & \\
\hline$>12$ & $583(74.6)$ & 70.8 & $\begin{array}{l}0.674(0.524- \\
0.866)\end{array}$ & 0.002 & $\begin{array}{l}0.579(0.448- \\
0.749)\end{array}$ & $\begin{array}{l}< \\
0.001\end{array}$ \\
\hline \multicolumn{7}{|l|}{ CEA level } \\
\hline Normal & $438(56.0)$ & 72.8 & 1 (ref) & & 1 (ref) & \\
\hline Elevated & $344(44.0)$ & 61.3 & $\begin{array}{l}1.462(1.158- \\
2.847)\end{array}$ & 0.001 & $\begin{array}{l}1.141(0.876- \\
1.486)\end{array}$ & 0.329 \\
\hline \multicolumn{7}{|l|}{ CA19-9 level } \\
\hline Normal & $636(81.3)$ & 70.8 & 1 (ref) & & 1 (ref) & \\
\hline Elevated & $136(18.7)$ & 54.8 & $\begin{array}{l}1.703(1.299- \\
2.232)\end{array}$ & $<.001$ & $\begin{array}{l}1.514(1.130- \\
2.027)\end{array}$ & 0.005 \\
\hline \multicolumn{7}{|c|}{ Surgical procedure } \\
\hline laparoscopic & $597(76.3)$ & 69.3 & 1 (ref) & & 1 (ref) & \\
\hline Open & $185(23.7)$ & 62.7 & $\begin{array}{l}1.356(1.044- \\
1.760)\end{array}$ & 0.022 & $\begin{array}{l}1.223(0.934- \\
1.602)\end{array}$ & 0.144 \\
\hline \multicolumn{7}{|l|}{$\begin{array}{l}\text { Postoperative } \\
\text { complications }\end{array}$} \\
\hline No & $723(92.5)$ & 70.3 & 1 (ref) & & 1 (ref) & \\
\hline Yes & $59(7.5)$ & 37.3 & $\begin{array}{l}2.385(1.687- \\
2 .=3.373)\end{array}$ & $<.001$ & $\begin{array}{l}1.815(1.247- \\
2.642)\end{array}$ & 0.002 \\
\hline \multicolumn{7}{|l|}{$\begin{array}{l}\text { Postoperative } \\
\text { chemotherapy }\end{array}$} \\
\hline Absent & $181(23.1)$ & 59.1 & 1 (ref) & & 1 (ref) & \\
\hline Present & $601(76.9)$ & 70.4 & $\begin{array}{l}0.639(0.494- \\
0.826)\end{array}$ & 0.001 & $\begin{array}{l}0.667(0.505- \\
0.880)\end{array}$ & 0.004 \\
\hline
\end{tabular}

\section{Patients characteristics and survival after propensity score matching}

We analyzed 286 patients in each group, who were selected by one-to-one matching using propensity scores. The median follow-up was 66 (range: 6-105) months for the matched pair. Characteristics after the propensity score analysis are shown in the right columns of Table 2. Two hundred eighty-six of the 496 patients in the LG were matched with 286 patients in the SG after covariate adjustment. The adjusted propensity score for patients in the LG was approximately identical to that for patients in the SG $(0.425 \pm 0.103 v s 0.425 \pm 0.098, P$ 
$=0.927$ ). Figure 2 displays the distribution of the propensity scores in the matched and unmatched patients in the $L G$ and those in the SG. All covariates were equally distributed over the two matched groups. Matched patients in the LG had a similar sex ratio, mean age at surgery, tumor location, histology, lymphovascular invasion, number of lymph nodes retrieval, lymph node metastasis, CEA level, CA19-9 level, surgical procedure, postoperative complications and postoperative chemotherapy, as those of the matched patients in the SG.

In the matched study series, patients with larger tumors still had a lesser 5-year OS than those with smaller tumors (5-year OS: $58.7 \%$ vs $75.2 \%, P<0.001$, Fig. 1B).

\section{Recurrence data of the whole study series}

The patterns and incidence of recurrence are shown in Table 4. Although there were no significant differences in locoregional recurrence between the two groups, patients with larger tumors had a significantly higher overall recurrence rate than those with smaller tumors $(41.1 \%$ vs $27.6 \%, P<0.001)$. The incidence of distant metastasis was greater in patients with larger tumors as well $(37.1 \%$ vs $25.2 \%, P=0.001)$. For patients with distant metastasis, liver $(19.2 \%$ vs $12.9 \%, P=0.025)$, lung $(9.9 \%$ vs $5.6 \%, P=0.037)$ and peritoneal $(6.0 \%$ vs $2.8 \%, P=0.042)$ metastasis were still more common in those with larger tumors than those with smaller tumors, and the difference was statistically significant.

Table 4

Type of initial recurrence in the whole study series

\begin{tabular}{|llll|}
\hline Type of recurrence & $\mathbf{S G}(\mathbf{n = 2 8 6})$ & $\mathbf{L G}(\mathbf{n}=\mathbf{4 9 6})$ & $\boldsymbol{P}$ \\
\hline Locoregional & $7(2.4)$ & $20(4.0)$ & 0.242 \\
\hline Distant metastasis & $72(25.2)$ & $184(37.1)$ & 0.001 \\
\hline Liver & $37(12.9)$ & $95(19.2)$ & 0.025 \\
\hline Lung & $16(5.6)$ & $49(9.9)$ & 0.037 \\
\hline Peritoneal & $8(2.8)$ & $30(6.0)$ & 0.042 \\
Others & $11(3.8)$ & $10(2.0)$ & 0.127 \\
Overall recurrence & $79(27.6)$ & $204(41.1)$ & $<0.001$ \\
\hline
\end{tabular}

\section{Tumor size as an indicator for good response of postoperative chemotherapy}

In the multivariate analysis, postoperative chemotherapy was an independent prognostic factor for OS.

Patients receiving postoperative adjuvant chemotherapy had a significantly higher OS rate than those without chemotherapy (5-year OS: $70.4 \%$ vs $59.1 \%, P=0.001$ ). The proportion of receiving postoperative chemotherapy in patients with smaller tumors was almost equal to that in patients with larger tumors $(74.1 \%$ vs $78.4 \%, P=0.170)$. Stratified analysis of tumor size revealed that in the $\mathrm{SG}$, the 5 -year OS rates were $78.3 \%$ and $66.2 \%$ for patients with and without chemotherapy $\left(X^{2}=6.027, P=0.014\right)$, respectively; and in the $L G$, the 5 -year OS rates were $66.1 \%$ and $54.2 \%$ for patients with and without chemotherapy $\left(\chi^{2}=8.601, P=0.003\right)$, respectively (Fig. 3). Patients with larger tumors may benefit more from postoperative chemotherapy. 


\section{Incorporation of the tumor size into the eighth edition UICC TNM staging system}

The OS of N0-stage patients with larger tumors was similar to that of N1-stage patients with smaller tumors. The OS of N1-stage patients with larger tumors was equal to that of N2-stage patients with smaller tumors (Fig. 4). Based on these results, we established a newly modified tumor-size-node-metastasis (mTSNM) staging system by incorporation of the tumor size into the eighth edition of UICC TNM staging system. The new mTSNM staging system was presented in Table 5. For T4 stage CC patients without distant metastasis, the mTSNM stages were defined as follows: mIIB, NO-stage patients with smaller tumors; mIIIA, NO-stage patients with larger tumors and N1-stage pateints with smaller tumors; mllIB, N1-stage patients with larger tumors and N2-stage pateints with smaller tumors; and mIIIC, N2-stage pateints with larger tumors.

Table 5

T4 CC patients were divided into two groups according to the tumor size: S1 and S2 groups. S was included into staging system, then the new stages were suggested.

\begin{tabular}{|llll|}
\hline S & N0 & N1 & N2 \\
\hline S1 & IIB & IIIA & IIIB \\
\hline S2 & IIIA & IIIB & IIIC \\
\hline \multicolumn{4}{l}{ S: tumor size } \\
\hline
\end{tabular}

The prognostic value of the TNM stage and MTSNM stage were evaluated by univariate and multivariate analyses. In the TNM stages, the 5-year OS rates were $73.5 \%, 59.0 \%$ and $32.9 \%$ in the IIB, IIIB and IIIC stages, respectively $\left(X^{2}=95.542, P<0.001\right)$. In the mTSNM stages, the 5 -year OS rates were $81.9 \%, 71.0 \%, 51.3 \%$ and $26.4 \%$ in the mIIB, mIIIA, mIIIB and mIIIC stages, respectively $\left(X^{2}=120.375, P<0.001\right.$; Table 6, Fig. 5A, 5B). As presented in Fig. 6 , the largest subgroup in the TNM stage is IIB, wherase the largest subgroup in the mTSNM stage is IIIA. The differences in prognostic prediction between the eighth edition of the TNM stage and the mTSNM stage were compared directly. The MTSNM stage was confirmed to be a more accurate prognostic classification for predicting the OS of T4 stage CC patients after curative resection than the eighth edition of TNM stage. The -2 log likelihood of the mTSNM stage was 3469.212, which was less than the value of the TNM stage (3477.452). 
Table 6

Survival analysis of the 782 CC patients according to the TNM and mTSNM stages

\begin{tabular}{|c|c|c|c|c|c|c|c|}
\hline \multirow[t]{2}{*}{ Characteristics } & \multirow[t]{2}{*}{ Cases } & \multirow[t]{2}{*}{$\begin{array}{l}\text { 5-year } \\
\text { OS (\%) }\end{array}$} & \multicolumn{2}{|c|}{$\begin{array}{l}\text { Univariate } \\
\text { analysis }\end{array}$} & \multicolumn{2}{|c|}{ Multivariate analysis } & \multirow[t]{2}{*}{-2loglikelihood } \\
\hline & & & $x^{2}$ & $\mathbf{P}$ & $\mathrm{HR}(95 \% \mathrm{Cl})$ & p & \\
\hline $\begin{array}{l}\text { TNM stage } \\
\text { (T4M0) }\end{array}$ & & & 95.542 & $<.001$ & $\begin{array}{l}1.512(1.374- \\
1.663)\end{array}$ & $\begin{array}{l}< \\
0.001\end{array}$ & 3477.452 \\
\hline IIB(N0) & 547 & 75.3 & & & & & \\
\hline IIIB(N1) & 156 & 59.0 & & & & & \\
\hline IIIC(N2) & 79 & 32.9 & & & & & \\
\hline $\begin{array}{l}\text { mTSNM stage } \\
\text { (T4M0) }\end{array}$ & & & 120.375 & $<.001$ & $\begin{array}{l}1.939(1.700- \\
2.212)\end{array}$ & $\begin{array}{l}< \\
0.001\end{array}$ & 3469.212 \\
\hline IIB(S1N0) & 193 & 81.9 & & & & & \\
\hline IIIA(S1N1/S2N0) & 421 & 71.0 & & & & & \\
\hline IIIB(S1N2/S2N1) & 115 & 51.3 & & & & & \\
\hline IIIC(S2N2) & 53 & 26.4 & & & & & \\
\hline
\end{tabular}

\section{Discussion}

Tumor size, given as the maximum diameter of the tumor, was one of independent prognostic factors of many solid tumors ${ }^{1-5}$. As for gastrointestinal carcinoma, several studies ${ }^{6-9}$ affirmed that tumor size positively correlated with important prognostic factors and negatively impacted survival. However, comparing with depth of invasion, tumor size is not a better predictive factor, and its prognostic value is often neglected. Some researchers believed that tumor size was easily affected by many other factors including depth of invasion and lymph node metastasis, and it could not predict prognosis independently. In addition, it was difficult to reach consensus on the best cutoff points of tumor size worldwide ${ }^{17-18}$. At present, the prognostic value of tumor size in CC remains controversial. In this study, we found that patients with larger tumors and patients with smaller tumors were extremely imbalanced in basic clinicopathological factors. To eliminate bias due to different distribution of covariates for the groups, propensity score matching method and multivariate Cox regression analysis were applied together. It was confirmed that tumor size was one of independent prognostic factors and larger tumor size was associated with higher frequency of distant metastasis and better response of postoperative chemotherapy. Incorporation of tumor size into the eighth edition of TNM staging system could improve the accuracy of the prognostic prediction of T4 stage CC patients.

Tumor size can be objectively and easily measured. Its prognostic value and clinical significance have been widely evaluated in gastric cancer. Several studies ${ }^{19-21}$ confirmed that larger tumor size was associated with significantly poorer OS than smaller tumor size in a given subset of gastric cancer, such as Borrmann type III, node-negative, or T4aNOMO stage disease. As digestive tract tumors, CC and gastric cancer have some similar 
clinicopathological features. Besides, tumor size is closely related to surgical methods and scope of CC. The prognostic value of tumor size in CC should be explored as well. Theoretically, tumor size increases with tumor progression, and patients with larger tumors usually have a poorer prognosis than those with smaller ones. Previous studies ${ }^{6-9}$ had confirmed this theory and concluded that poorer prognosis of larger tumors was associated with tumor necrosis, iron deficiency anemia, tumor grade and a more aggressive underlying biology. In contrast, some studies ${ }^{10-12,15,16}$ reveled that patients with smaller tumors had a worse prognosis in a given subset of CC, such as positive lymph node and IIA stage disease. In these studies ${ }^{10,13-15}$, the researchers considered that the poor prognosis of CC patients with smaller tumors in the same stage was related to a more biologically aggressive phenotype. In addition, surgeons are more likely to treat larger tumors more aggressively by extending lymph node dissection or postoperative chemotherapy, which may account for the better survival of patients with larger tumors ${ }^{15}$. As tumor size is associated with lymph node metastasis, depth of invasion and other prognostic factors, it is crucial to balance the relevant factors in both groups. To the best of our knowledge, this study is the first analysis using propensity score matching methods to assess the impact of tumor size on the prognosis of CC patients. We found that the incidence of lymphovascular infiltration, undifferentiated type and postoperative complications were higher in patients with larger tumors. Besides, patients with larger tumors had a significant lower 5-year OS rate than those with smaller tumors and tumor size was an independent prognostic factor of CC patients. The results were consistent with previous studies $^{8-9}$. After matching, patients with larger tumors still demonstrated a significant lower 5-year OS rate than those with smaller tumors $(63.5 \%$ versus $74.2 \%, P<0.001)$. Although previous studies have reported some possible causes of the prognostic impact of tumor size, so far, the mechanism remains unclear. Usually, the direction of primary tumor infiltration includes along the intestinal wall and perpendicular to the intestinal wall. The former forms tumor size, while the latter contributes to depth of invasion. For tumors at the same T stage, the prognosis of patients with larger tumors is worse than that of patients with smaller tumors, which may be due to the larger tumor burden and more likely to invade vascular and lymphatic channels in larger tumors. In addition, larger tumors were associated with significant reductions in serum hemoglobin and albumin, but increased chances of bowel obstruction. These factors have been affirmed to be associated with poor prognosis of $\mathrm{CC}$ in previous studies ${ }^{22-25}$. We believe that the depth of tumor invasion plays a major role in the prognosis of $\mathrm{CC}$, but the effect of tumor size on survival can not be ignored.

Actually, in the present study we confirmed that tumor size was not only associated with postoperative recurrence, but also a good indication of postoperative chemotherapy. Patients with larger tumors had a significantly higher overall recurrence rate, especially distant metastasis than those with smaller tumors. Previous studies ${ }^{26-28}$ confirmed that the possibility of lymphovascular invasion increased with the increase of tumor size, which might account for higher recurrence rate of larger tumor size. We believe that tumor cells that invade lymphovascular eventually form distant metastases and larger tumor size should be regarded as a high risk factor for recurrence. CC patients with larger tumor size should be closely followed up. Besides, postoperative chemotherapy should be considered in these patients even without lymph node metastasis. In fact, these patients do benefit more from chemotherapy in the present study.

Tumor size, as a T staging of many solid tumors, has been incorporated into the TNM staging system. As for digestive tract tumors, the depth of invasion plays a greater role in prognosis than the tumor size, so it is

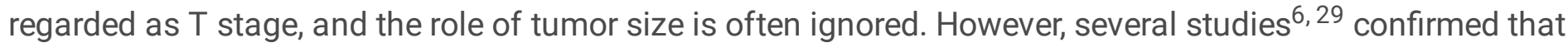


incorporation of tumor size into the staging system could improve the prognostic prediction of gastric cancer. Deng et al. ${ }^{6}$ even used tumor size as a T classification and established a new tumor size-node-metastasis classification system. They found that the new tumor size-node-metastasis classification could accurately evaluate prognosis and provide very powerful discrimination of patients' OS, as compared with TNM classification. Until now, few studies have incorporated tumor size into the staging system of colon cancer. In the present study, we found that the OS of NO-stage patients with larger tumors was similar to that of N1-stage patients with smaller tumors and the OS of N1-stage patients with larger tumors was equal to that of N2-stage patients with smaller tumors. Based on the results, we incorporated tumor size into the TNM staging system and established a new mTSNM classification. It was affirmed that the mTSNM classification was a more appropriate prognostic classification to predict the OS of CC patients than the eighth edition of the TNM staging system. We believed that the current edition of the TNM staging system had following shortcomings. Firstly, it could not reflect the continuity of tumor progression. For example, the stage of T4NOMO patients is IIB, however, it crosses IIIA stage and jumps to IIIB and IIIC stages once lymph nodes are involved. Besides, the IIIA stage merely includes T1N1-2aM0 and T2N1M0 patients, however, lymph node metastasis is rare in T1-2 stage patients. In our suggested mTSNM staging system, patients were continuously and uniformly distributed from IIB to IIIC stage and the largest subgroup was IIIA stage.

There are several limitations to our study. First and foremost are the limitations inherent to retrospective analyses. Moreover, as the sample size was relatively small, patients was simply divided into two groups based on best cutoff value of tumor size, more elaborate division of subgroups was not performed. Optimal cut-off values vary among different parts of the large bowel, usually decreasing from the right colon to the left, while tumor location was not concerned when identifying best threshold. Nevertheless, even with these limitations our results suggested that tumor size is relevant in patients with colon cancer.

\section{Conclusion}

Tumor size is an independent prognostic factor and negatively impacts survival of CC patients. Larger tumor size could correlate with a higher distant metastasis rate and better response of adjuvant chemotherapy. Prognostic impact of tumor size should be considered when making adjuvant therapy decisions and prognosis evaluation. Besides, we maintain that tumor size should be incorporated into the staging system to enhance the accuracy of the prognostic prediction of T4 stage CC patients. Further studies are necessary to elucidate the mechanism of tumor size as a prognostic factor in CC.

\section{Declarations}

\section{Competing interests}

The authors declare no competing interests.

\section{Acknowledgements}

This study was supported in part by grants from the Key Projects of Research and Development of Science and Technology Department of Hainan Province (No. ZDYF2020137) 


\section{References}

1. Wei W. et al. Comparison of Residual Risk-Based Eligibility vs Tumor Size and Nodal Status for Power Estimates in Adjuvant Trials of Breast Cancer Therapies. JAMA Oncol. 4, e175092(2018).

2. Cortesi L. et al. Tumor size, node status, grading, HER2 and estrogen receptor status still retain a strong value in patients with operable breast cancer diagnosed in recent years. Int J Cancer. 132, E58-65 ( 2013)

3. Pang Z. et al. Prognostic effects of preoperative obstructive pneumonitis or atelectasis and comparison with tumor size in non-small cell lung cancer. J Thorac Dis. 9, 768-778 (2017).

4. Travis W.D. et al. The IASLC Lung Cancer Staging Project: Proposals for Coding T Categories for Subsolid Nodules and Assessment of Tumor Size in Part-Solid Tumors in the Forthcoming Eighth Edition of the TNM Classification of Lung Cancer. J Thorac Oncol. 11, 1204-1223 (2016).

5. Hwang S. et al. The Impact of Tumor Size on Long-Term Survival Outcomes After Resection of Solitary Hepatocellular Carcinoma: Single-Institution Experience with 2558 Patients. J Gastrointest Surg. 19, 12811290 (2015).

6. Deng J. et al. Tumor size as a recommendable variable for accuracy of the prognostic prediction of gastric cancer: a retrospective analysis of 1,521 patients. Ann Surg Oncol. 22, 565-572 (2015).

7. Feng H. et al. Association of tumor size with prognosis in colon cancer: A Surveillance, Epidemiology, and End Results (SEER) database analysis. Surgery. https//:doi: 10.1016/j.surg.2020.11.011 (2020).

8. Saha S. et al. Tumor size predicts long-term survival in colon cancer: an analysis of the National Cancer Data Base. Am J Surg. 209, 570-574 (2015).

9. Dai W. et al. The Critical Role of Tumor Size in Predicting Prognosis for T1 Colon Cancer. Oncologist. 25, 244-251 (2020).

10. Lee S.Y. et al. Macroscopic serosal invasion and small tumor size as independent prognostic factors in stage IIA colon cancer. Int J Colorectal Dis. 33, 1139-1142 (2018).

11. Wang Y. et al. Unfavorable effect of small tumor size on cause-specific survival in stage IIA colon cancer, a SEER-based study. Int J Colorectal Dis. 30, 131-137 (2015).

12. Santullo F. et al. Tumor size as a prognostic factor in patients with stage lla colon cancer. Am J Surg. $\mathbf{2 1 5}$, 71-77 (2018).

13. Huang B. et al. Smaller tumor size is associated with poor survival in T4b colon cancer. World $\mathrm{J}$ Gastroenterol. 22, 6726-6735 (2016).

14. Yamanashi T. et al. Laparoscopic surgery for locally advanced T4 colon cancer: the long-term outcomes and prognostic factors. Surg Today. 48, 534-544 (2018).

15. Huang B. et al. Smaller tumor size is associated with poor survival in stage II colon cancer: An analysis of 7,719 patients in the SEER database. Int J Surg. 33, 157-163 (2016).

16. Muralidhar V. et al. Association Between Very Small Tumor Size and Increased Cancer-Specific Mortality in Node-Positive Colon Cancer. Dis Colon Rectum. 59, 187-193 (2016).

17. Kooby D.A. et al. Biologic predictors of survival in node-negative gastric cancer. Ann Surg. 237, 828-837 (2003). 
18. Huang K.H. et al. Factors affecting recurrence in node-negative advanced gastric cancer. J Gastroenterol Hepatol. 24, 1522-1526 (2009).

19. Hosoda K. et al. Preoperative tumor size is a critical prognostic factor for patients with Borrmann type III gastric cancer. Surg Today. 45, 68-77 (2015).

20. Quan J. et al. The impact of tumor size on survival of patients with pT4aNOMO gastric cancer. Am Surg.79, 328-331 (2013).

21. Zhao LY. et al. A new predictive model combined of tumor size, lymph nodes count and lymphovascular invasion for survival prognosis in patients with lymph node-negative gastric cancer. Oncotarget. 7, 7230072310 (2016).

22. Chin C.C. et al. Carcinoma obstruction of the proximal colon cancer and long-term prognosis--obstruction is a predictor of worse outcome in TNM stage II tumor. Int J Colorectal Dis. 25, 817-822 (2010).

23. Li F. et al. Serum iron and ferritin levels in patients with colorectal cancer in relation to the size, site, and disease stage of cancer. J Gastroenterol. 34, 195-199, (1999).

24. Fujikawa H. et al. Prognostic Impact of Preoperative Albumin-to-Globulin Ratio in Patients with Colon Cancer Undergoing Surgery with Curative Intent. Anticancer Res. 37, 1335-1342 (2017).

25. Boonpipattanapong T. et al. Preoperative carcinoembryonic antigen and albumin in predicting survival in patients with colon and rectal carcinomas. J Clin Gastroenterol. 40, 592-595 (2006)

26. Seijong Kim. et al. Lymphovascular invasion, perineural invasion, and tumor budding are prognostic factors for stage I colon cancer recurrence. Int J Colorectal Dis. 35, 881-885 (2020).

27. Rössler O. et al. Tumor size, tumor location, and antitumor inflammatory response are associated with lymph node size in colorectal cancer patients. Mod Pathol. 30, 897-904 (2017).

28. Huh J.W. et al. Prognostic significance of lymphovascular or perineural invasion in patients with locally advanced colorectal cancer. Am J Surg.206, 758-763 (2013).

29. Liang Y. et al. Tumor Size Improves the Accuracy of the Prognostic Prediction of Lymph Node-Negative Gastric Cancer. J Surg Res. 240, 89-96 (2019).

\section{Figures}
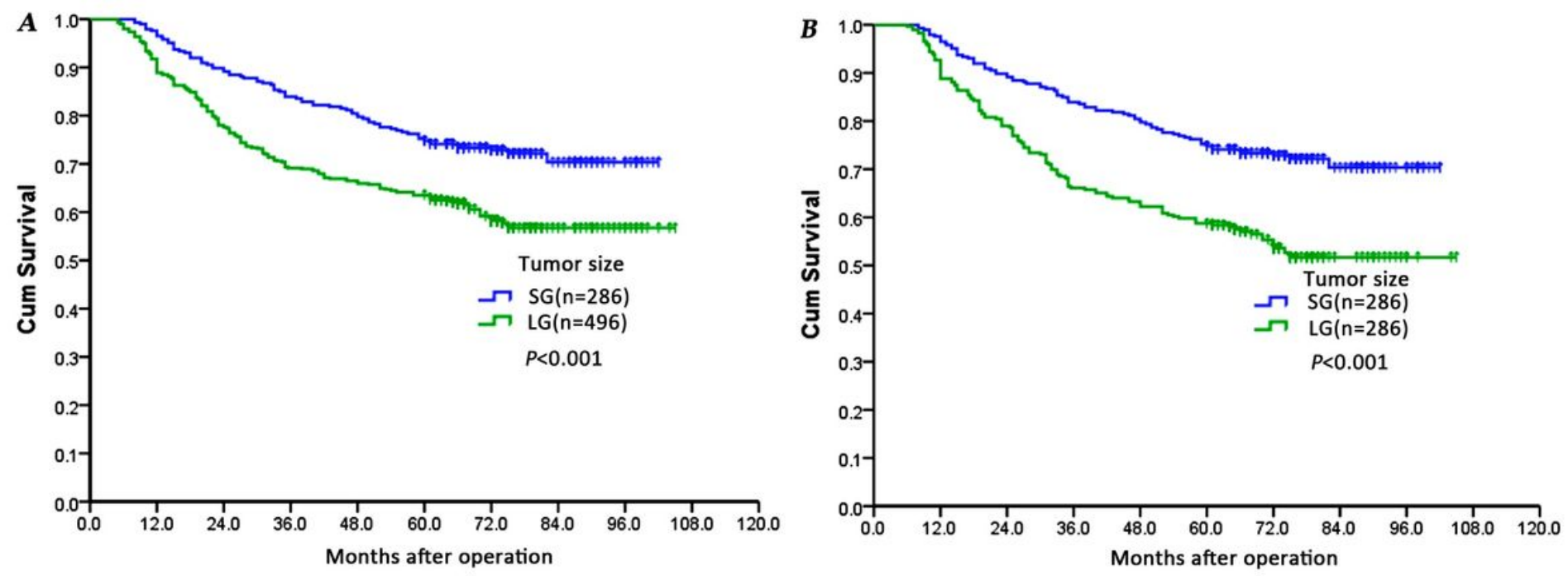


\section{Figure 1}

Prognosis of CC patients who underwent curative surgery. Pateints were categorized into two groups according to the tumor size: SG and LG. (A) Survival curve for all patients: the 5 -year OS rates were $75.2 \%$ and $65.3 \%$ for $S G$ and $L G$, respectively $(P<0.001)$. (B) Survival curve for matched patients: the 5 -year $0 S$ rates were $75.2 \%$ and $58.7 \%$ for $S G$ and $L G$, respectively $(P<0.001)$.

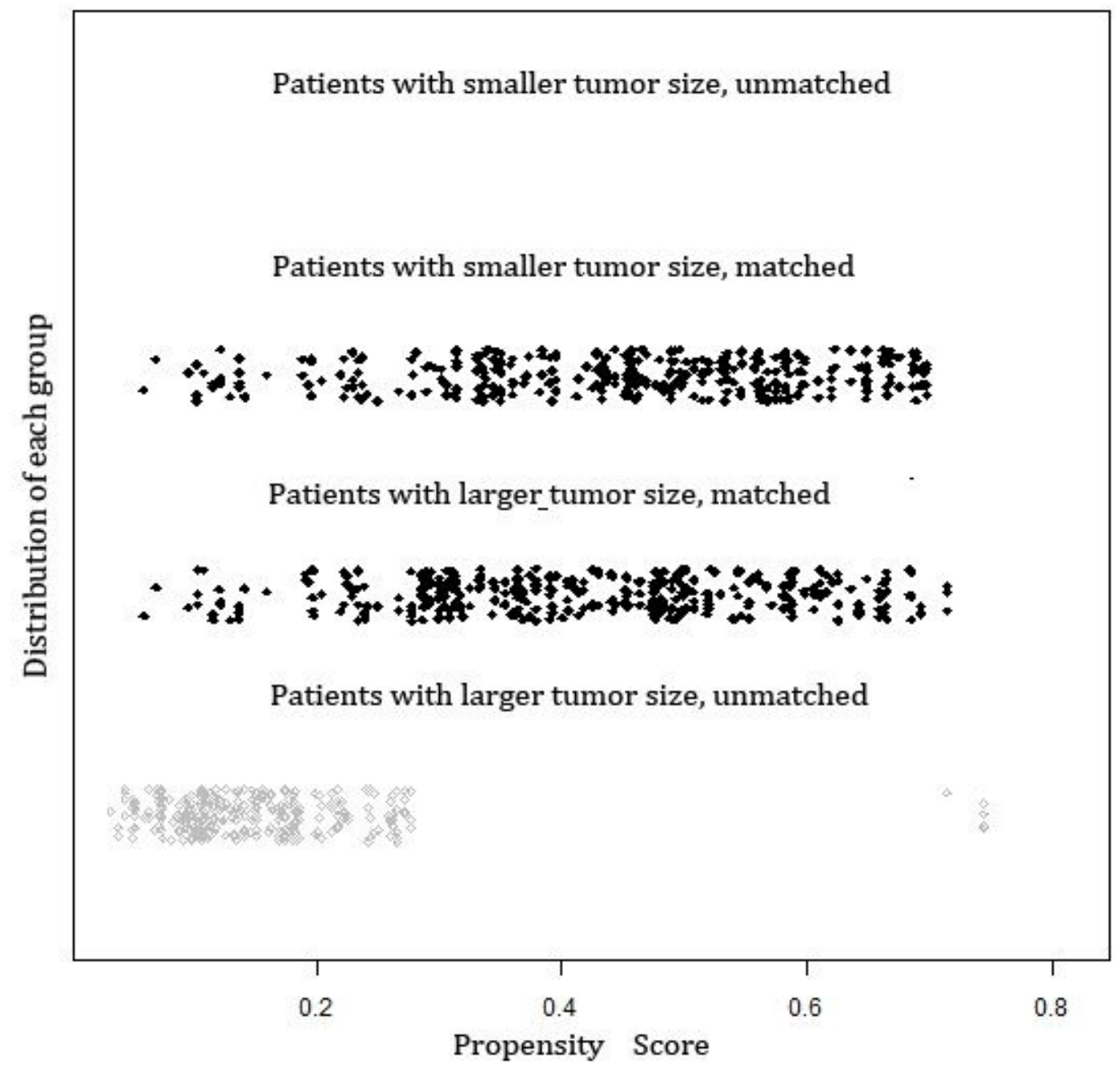

Figure 2

Distribution of the propensity scores. Each circle represents one patients. 

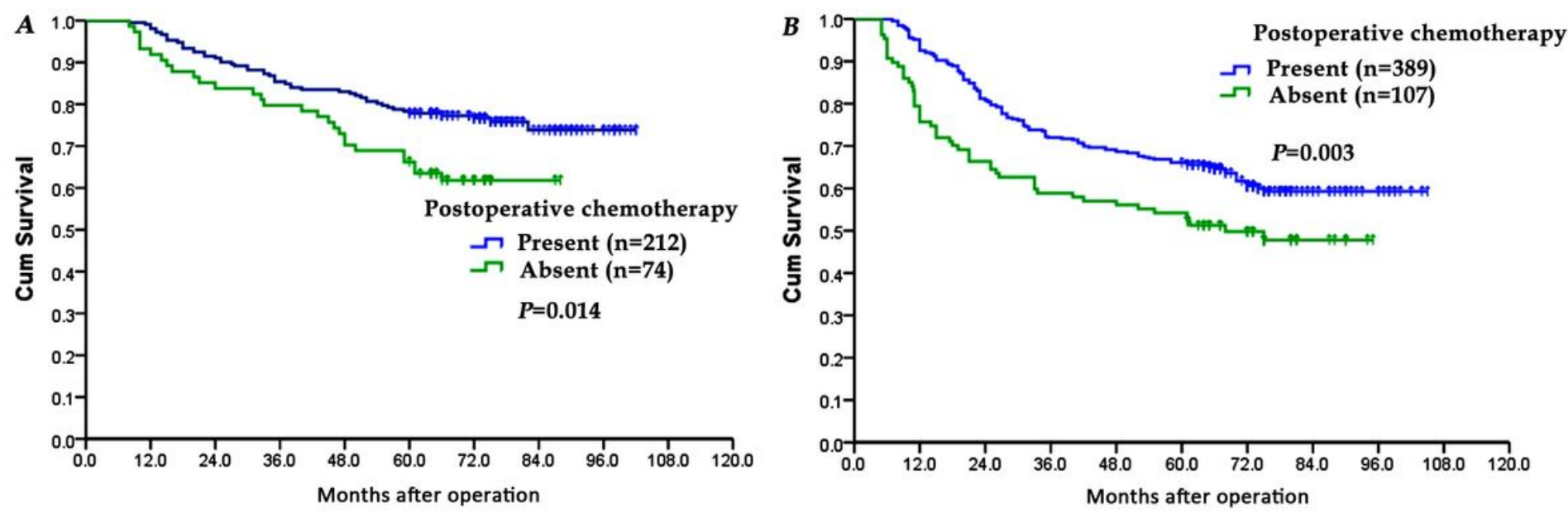

Figure 3

Survival curves of all patients categorized by postoperative chemotherapy. (A) Patients with smaller tumors: the 5 -year OS rates were $78.3 \%$ and $66.2 \%$ for patients with and without chemotherapy (c2=6.027, $P=0.014$ ). (B) Patients with larger tumors: the 5-year OS rates were $66.1 \%$ and $54.2 \%$ for patients with and without chemotherapy (c2=8.601, $\mathrm{P}=0.003)$.

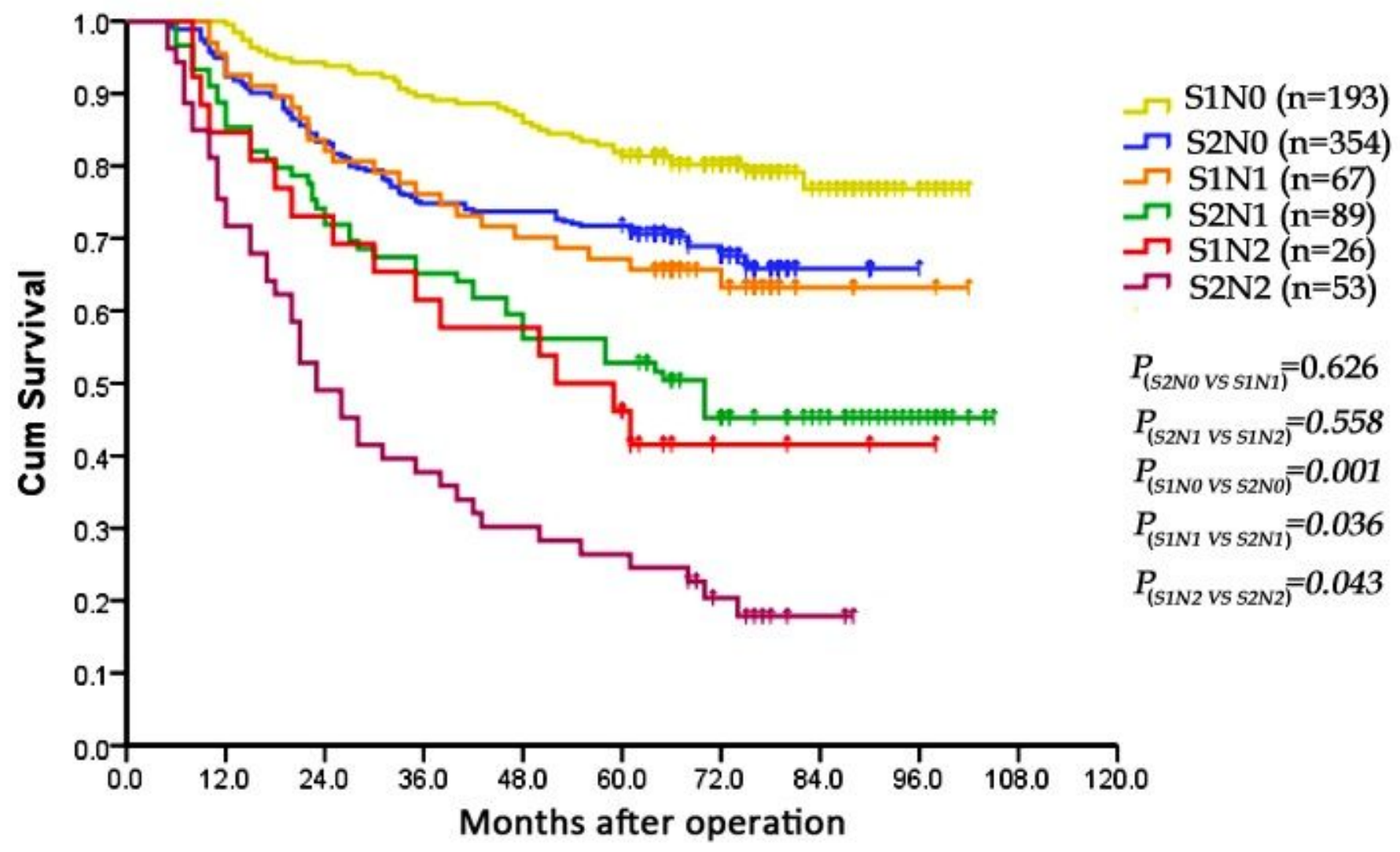

Figure 4 
Comparison of survival curves of T4M0 stage patients with different $\mathrm{N}$ stages and tumor sizes. The OS of NOstage patients with S2-size was similar to that of N1-stage patients with S1-size $(P=0.626)$. The OS of N1stage patients with S2-size was equal to that of N2-stage patients with S2-size ( $P=0.558)$.
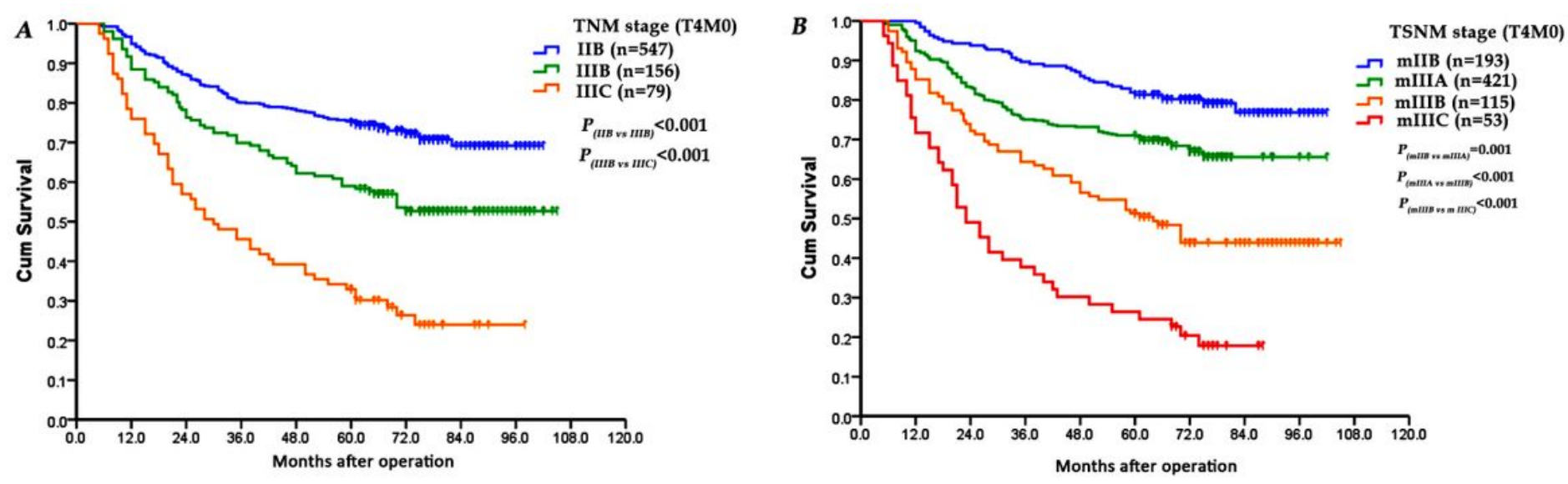

\section{Figure 5}

Survival curves of patients according to different tumor stages. (A) TNM stage $(P<0.001)$. (B) mTSNM stage $(\mathrm{P}<0.001)$.

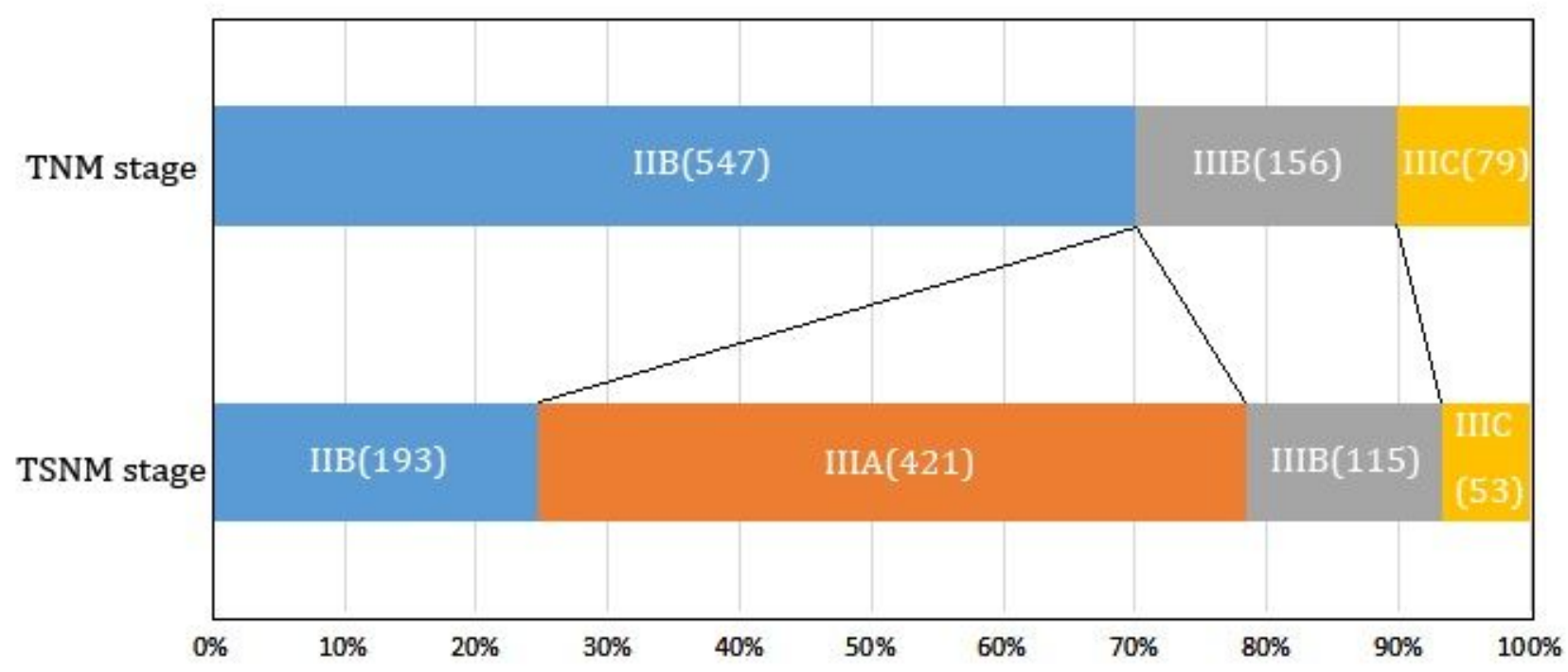

\section{Figure 6}

Patients distribution of different classification system. In the TNM staging system, patients were staged from IIB to IIIC, and the largest subgroup was IIB stage, however, there was no IIIA stage. In the MTSNM staging system, patients were continuously distributed from IIB to IIIC stage and the largest subgroup was IIIA stage. 